\title{
Disorders of the blood-aqueous barrier after phacoemulsification in diabetic patients
}

\begin{abstract}
Purpose To evaluate the function of the blood-aqueous barrier after phacoemulsification with implantation of a foldable intraocular lens (IOL) in diabetic patients.
\end{abstract}

Methods All patients were enrolled from those scheduled for phacoemulsification with intraocular lens implantation in Zhongshan Ophthalmic Center Guangzhou from March 2002 to June 2002. The classification on diabetic retinopathy (DR) was based on the fundus examination after cataract surgery. The blood-aqueous barrier function was examined using the laser flare cell meter (Kowa FC-2000) preoperatively and on postoperative days 1, 7, 30, and 90 by an independent examiner who was masked to the DR classification. Patients were operated by one experienced surgeon as per standard clinical protocol and were provided the same postoperative medical care. A linear regression and Wilcoxon test were used for the analysis.

Results A total of 112 patients were divided into three groups: patients without diabetic mellitus as normal control $(n=56)$, diabetic patients without diabetic retinopathy $(n=2)$, with nonproliferation diabetic retinopathy (NPDR) $(n=37)$, and diabetic patients with proliferation diabetic retinopathy (PDR) $(n=17)$. All patients were examined and successfully followed up for 3 months after cataract surgery. Aqueous flare mean photon counts in PDR, NPDR, and control eyes were $8.94 \pm 0.57,7.03 \pm 0.27$, and $6.94 \pm 0.34$ before surgery and increased to $32.42 \pm 0.67$, $26.07 \pm 0.83,26.27 \pm 1.37$ on the first day after surgery $(P<0.05)$, then decreased to $19.86 \pm 0.78,14.08 \pm 0.54$ and $13.96 \pm 1.05$ at 7 days after surgery $(P<0.05), 13.24 \pm 0.29$,
$9.86 \pm 0.33$, and $9.07 \pm 0.43$ at 30 days after surgery $(P<0.05)$; eventually, the counting decreased to $11.25 \pm 0.31,7.24 \pm 0.67$, and $7.16 \pm 0.27$ at 90 days after surgery $(P<0.05)$. Linear regression model suggested that other potential variables, such as age, sex, eye (left/ right), phaco time, phaco energy, and hypertension were not related to the outcome. For patients without diabetes mellitus and diabetic patients with NPDR, highly statistically significant differences $(P<0.05)$ were found between preoperative flare value and those measured on days 1,7 , and 30 after surgery, but no statistically significant differences $(P>0.05)$ were found between the preoperative flare value and those measured on postoperative days 90 . However, patients with PDR still had a higher flare value even on postoperative day 90 . The patients with intraoperative iris prolapse had a higher flare value between days 1 and 7 postoperatively. Conclusion Phacoemulsification with a foldable intraocular lens implantation affects the blood-aqueous barrier more severely in diabetic patients with PDR than patients with NPDR and nondiabetic patients.

Eye (2004) 18, 900-904. doi:10.1038/sj.eye.6701349 Published online 12 March 2004

Keywords: blood-aqueous barrier; phacoemulsification; lens implantation; intraocular; diabetes mellitus

Introduction

The recovery of the blood-aqueous barrier (BAB) after cataract surgery has been studied recently. Sanders et al ${ }^{1}$ reported the recovery of the $\mathrm{BAB}$ within 3 months after extracapsular $\mathrm{C}$ ataract surgery with intraocular lens (IOL) 
implantation by anterior chamber fluorophotometry. Other authors ${ }^{2,3}$ also described recovery within the same time after phacoemulsification using the laser flare cell meter (LFCM). However, Schauersberger et al ${ }^{4}$ reported a persistent $\mathrm{BAB}$ disorder even several years after cataract surgery.

The LFCM allows quantification of aqueous protein by computer analysis of light scattered by a helium neon beam projected into the anterior chamber. At the beginning of the 1990s, researchers started to use the LFCM to examine BAB disorders by measuring aqueous flare and cells. A statistically highly significant linear correlation between flare value and human albumin concentration in the anterior chamber was found, ${ }^{5}$ which suggests that the flare value could indirectly reflect the function of BAB. The immediate postoperative inflammation, stimulated by surgical invasion of the anterior uvea system, can be reduced by improvement in the surgical technique and better IOL design. ${ }^{6}$ However, there are few published data available on the changes in aqueous flare after cataract surgery in diabetic patients. Therefore, we examined flare value in the patients with age-related cataract and those with diabetes pre- and postoperatively.

\section{Patients and methods}

A total of 112 consecutive patients scheduled for cataract surgery (March 2002-June, 2002) who were attending the cataract service of Zhongshan Ophthalmic Center Guangzhou were enrolled in this study. In order to facilitate compliance on the follow-up, only patients who lived within $400 \mathrm{k}$ to the study hospital were chosen in this study. Further differentiation on NPDR or PDR classification was determined by fundus examinations after cataract surgery. In total, 56 of them were diagnosed as having diabetic mellitus at enrolment, among which, 37 with NPDR, 17 with PDR, and two without any signs of diabetic retinopathy; the two cases without diabetic retinopathy were combined into the NPDR group because the number of cases was too few for meaningful analysis. The treatment of diabetes included those who were under treatment with either diet control only (4.7\%), oral antidiabetics $(64.06 \%)$, or insulin $(31.25 \%)$ for glucose control. Exclusion criteria were a history of intraocular surgery, uveitis, laser treatment, and other ocular commodities besides age-related cataract. Written informed consent was obtained for all participants. This study was granted ethical approval by The Ethical Review Committee of Zhongshan Ophthalmic Center and adhered to the provisions of the Declaration of Helsinki for research involving human subjects.

\section{Standard cataract procedure}

The foldable IOL used was the Alcon AcrySof (model SA60AT), which has a $6.0 \mathrm{~mm}$ optic, a single piece, and an overall lens length of $13.0 \mathrm{~mm}$. All surgery was performed by the same experienced surgeon (L.Y.Z). Topical anaesthesia was used. The surgical procedure consisted of a $3.2 \mathrm{~mm}$ temporal clear corneal incision, followed by the performance of a continuous curvilinear capsulorhexis. After hydrodissection and hydrodelineation, phacoemulsification was performed. Cortical remnants were removed by automatic irrigation/aspiration. A one-piece acrylic IOL (Alcon, AcrySof, SA60AT) was implanted in the bag. The viscoelastic material was then carefully removed by irrigation. No subconjunctival steroids were used at the end of the surgery. All patients received the same postoperative therapy: topical combined dexamethasone-tobramycin eyedrops four times daily for the first week, two times a day for the second week, and then stop unless signs for severe postoperative inflammation was presented. The pre- and postoperative clinical assessments were performed in all cases by the same single observer who was masked to the DR classification.

\section{Measurement of blood-aqueous barrier function}

Flare values were evaluated by the LFCM (Kowa FC-2000). Measurements were made without dilation preoperatively as well as postoperatively on days $1,7,30$, and 90. To avoid the introduction of bias, an average of the five sequential readings was taken provided that the background scatter was less than $15 \%$ on each individual scan. The measurements were conducted by one experienced examiner who was masked to the diabetic status.

\section{Statistical analysis}

Aqueous flare values had a non-normal distribution. The recovery of the $\mathrm{BAB}$ was therefore assessed by a comparison of postoperative flare measurements with preoperative values using the Wilcoxon test. The linear regression was used to assess the influence of the potential risk factors (age, sex, phaco time, phaco energy, hypertension, and diabetes mellitus with or without proliferation retinopathy) on the flare values. $P$-values of $<0.05$ were considered statistically significant.

\section{Results}

All surgery was uneventful. In all, $69.6 \%$ of patients without diabetics and $67.7 \%$ of diabetic patients with 
NPDR achieved a best-corrected visual acuity of $20 / 40$ or better at day 7 postoperatively. However, only $33.1 \%$ of diabetic patients with PDR achieved a best corrected visual acuity of $20 / 40$ or better. At day $90,98.7 \%$ of patients without diabetes and $96.2 \%$ of diabetic patients with NPDR had a vision of $20 / 40$ or better. Only $35.2 \%$ of diabetics with PDR had an acuity of $20 / 40$ or better. We observed that the poor visual outcome in diabetic patients was attributable to macular oedema or macular haemorrhage.

The aqueous flare values in diabetic patients with PDR were significantly higher than those in normal control and in diabetic patients with NPDR preoperatively and at different interval postoperatively (Wilcoxon, $F=203.4$, $P<0.05)$, while there were no significant differences between those in normal control and diabetic patients with NPDR (Wilcoxon, $F=203.4, P>0.05$ ). The postoperative flare values were highest on the first postoperative day declining rapidly in the first week. Highly statistically significant differences (Wilcoxon, $F=218.6 .4, P<0.05)$ were found between the preoperative flare value and those measured on days 1,7 , and 30 after surgery, but no statistically significant differences (Wilcoxon, $F=218.6, P>0.05$ ) were found between preoperative flare value and those measured at 90 days in patients without diabetes and diabetic patients with NPDR. Diabetic patients with NPDR had no influence on the outcome, whereas diabetic patients with PDR had a higher flare value pre- and postoperatively (Table 1). Furthermore, they still had a disorder of BAB on day 90 after surgery. Other variables such as age, sex, hypertension, phaco time, phaco energy, and phaco time $\times$ phaco energy had not correlated with the flare value. For the patients with intraoperative iris prolapse, aqueous flare values in PDR, NPDR, and control eyes increased to $37.13 \pm 0.49,30.04 \pm 0.61$, and $29.71 \pm 0.82$ on the first day after surgery $(P<0.05)$, then decreased to $24.14 \pm 0.69,17.08 \pm 0.39$, and $16.96 \pm 0.75$ at 7 days after surgery $(P<0.05)$. The patients with intraoperative iris prolapse had a higher flare value between days 1 and 7 postoperatively and after that, there was no statistically significant difference between the patients with intraoperative iris prolapse and those without intraoperative iris prolapse.

No posterior capsular rupture, no vitreous prolapse and other severe complications occurred in any case.

\section{Discussion}

The phenomena that products such as protein cannot easily infiltrate into aqueous humour from the vessel even if it has higher plasma protein concentration called $\mathrm{BAB}$. BAB are mainly composed of two parts, One is the tight junction between the ciliary body nonpigment epithelial cells, and the other is the tight junction between iris endothelial cells.

Any traumatic events in the eye, whether due to injury or surgery, results in a various extents of disturbance on the $\mathrm{BAB}$. An increase of the protein concentration in the aqueous is the most straightforward evidence for the

Table 1 Demographic, pre-operative and operation status

\begin{tabular}{|c|c|c|c|}
\hline & Cataract with NPDR & Cataract with PDR & Cataract control \\
\hline$N$ & 39 & 17 & 56 \\
\hline Age $($ Mean \pm SD) & $64.7 \pm 3.8$ & $59.4 \pm 5.1$ & $71.3 \pm 2.6$ \\
\hline Sex (\% of Male) & 47.37 & 42.86 & 44.44 \\
\hline \multicolumn{4}{|l|}{ Visual acuity } \\
\hline Preoperation & $\mathrm{HM} / 30 \mathrm{~cm}-0.3$ & $\mathrm{HM} / 30 \mathrm{~cm}-0.1$ & $\mathrm{HM} / 30 \mathrm{~cm}-0.4$ \\
\hline Postoperative day 1 & $0.23(0.1-1.0)$ & $0.12(\mathrm{FC} / 30 \mathrm{~cm}-0.2)$ & $0.47(0.2-1.0)$ \\
\hline Postoperative day 7 & $0.61(0.2-1.0)$ & $0.2(0.04-0.5)$ & $0.63(0.3-1.2)$ \\
\hline Postoperative day 30 & $0.69(0.4-1.0)$ & $0.28(0.1-0.5)$ & $0.76(0.4-1.2)$ \\
\hline Postoperative day 90 & $0.71(0.4-1.0)$ & $0.25(0.1-0.5)$ & $0.75(0.4-1.2)$ \\
\hline \multicolumn{4}{|l|}{ Lens nucleus grade (\%) } \\
\hline Grade 3 & 47.37 & 43.29 & 44.44 \\
\hline Grade 4 & 42.10 & 46.43 & 42.59 \\
\hline Grade 5 & 10.53 & 10.28 & 12.07 \\
\hline Blood pressure $(S B P / D B P)($ mean $\pm S D) m m H g$ & $137.1 \pm 1.24 / 76.18 \pm 1.02$ & $142.8 \pm 1.67 / 83.1 \pm 0.59$ & $135.9 \pm 1.13 / 79.46 \pm 0.81$ \\
\hline Blood glucose level (mean \pm SD)mmol/1 & $7.91 \pm 0.63$ & $10.8 \pm 0.57$ & $5.72 \pm 0.25$ \\
\hline Phaco time (mean \pm SD)second & $27.1 \pm 0.22$ & $.8 \pm 0.27$ & $27.6 \pm 0.12$ \\
\hline Phaco energy (mean $\pm S D$ ) & $14.87 \% \pm 0.41$ & $16.35 \% \pm 0.32$ & $14.69 \% \pm 0.38$ \\
\hline
\end{tabular}


breakdown of the BAB. Limited by the available examination technique, the protein concentration in the anterior chamber can only be estimated by slit-lamp microscopy. However, this measurement is semiquantitative, being unable to quantify the level of the protein concentration and detect the subtle changes at a reproducible pattern. Hence, the reading depends on the subjective judgement and experience of the examiner. On the other hand, aqueous humour obtained by anterior chamber paracentesis was invasive and potentially harmful, which was generally not acceptable for the patients. The advent LFCM equipment perhaps can provide a better clinical option and allows a noninvasive and quantitative evaluation of the BAB breakdown.

$\mathrm{BAB}$ disorders after cataract surgery have been reported previously. However, to our knowledge, few reports so far are available regarding the breakdown of BAB after phacoemulsification with one-piece foldable IOL implantation, particularly for the diabetic patients with PDR; few researches have been conducted to evaluate the disorders of $\mathrm{BAB}$ after cataract surgery by the LFCM in Chinese patients with dark brown thick irises and relatively hard lens nucleus. The flare intensity value in our group of patients was significantly lower than what was reported in European eyes, which might be attributable to shorter phaco time and a surgeon with a more sophisticated surgical technique.

While extracapsular cataract extraction with manual expression of the nucleus of the lens was considered a standard procedure for cataract surgery in the developing world up to now, a transition to a smallincision surgery with phacoemulsification is becoming more popular in order to ensure fewer disturbances on the anterior segment and achieve better vision outcome and early recovery. ${ }^{7}$ The improvement in the design and material of the IOL further enhances this advantage by improving the biological compatibility and therefore minimizing the postoperative immune reaction. ${ }^{6,8}$ However, the conclusion on how and when the BAB can recover was controversial. Our study highlights these characteristics in age-related cataract without diabetes mellitus and also compare the differences between patients with or without proliferation of diabetic retinopathy.

Diabetes mellitus is a complex metabolic disorder that also involves the small blood vessels, often causing widespread damage to many body tissues, including the eye. High level of blood glucose in the diabetic patients results in oedema of the iris capillary endothelium, which subsequently leads to the decrease of the resistance of the vessel wall and the increase of fragility. Theoretically, surgical trauma may more easily lead to disorders of the BAB in diabetic patients. This study evaluates the recovery of $\mathrm{BAB}$ in diabetic patients in comparison with patients without diabetes after phacoemulsification with foldable IOL implantation.

Data showed that aqueous flare values were greatest on the first postoperative day and then declined rapidly in the first week and gradually thereafter (Table 2).

Moreover, there was no statistically significant difference between them. Re-establishment of BAB function in most patients without diabetes and diabetes patients with NPDR was found to occur in a relatively short period following surgery. Our result is not consistent with the other studies. ${ }^{4}$ In their results, the re-establishment of $\mathrm{BAB}$ had a relatively longer period. The reasons for this may be related to different phaco time, different surgical technique, different IOL material, and other unknown causes. Furthermore, whether the single-piece foldable IOL results in less disorders of the BAB should be investigated later. ${ }^{9}$ In general, our study suggests that the small incision cataract surgery with the foldable IOL implantation is a safer and more effective treatment option for diabetic patients.

However, the flare values in diabetic patients with proliferation retinopathy examined preoperatively and at days $1,7,30$, and even at 90 were significantly higher than those of the patients without diabetes mellitus or diabetic patients with nonproliferation retinopathy. The function of the BAB in patients with proliferation retinopathy did not completely recover when we followed up on them at 3 months after surgery. The mechanism for the repair of BAB function after surgery might be different in PDR patients. This raises the question as to whether the degree of defect on the iris microcirculation in patients with PDR occurring in the same pattern as the retinal or iris microcirculation, and needs to be further investigated.

Most of the patients (without diabetes and diabetes with NPDR) had a best-corrected vision less than 20/40 on the first postoperative day. We think that the main reason for this is corneal oedema (because of the harder lens nucleus). With the attenuation of the oedema, the best-corrected vision improved rapidly. However, most of the patients with PDR had a vision less than 20/40 till day 90 following surgery. Those patients had retinopathy

Table 2 Mean photon count/ms in age-related cataract and diabetic cataract (mean $\pm \mathrm{SD})$

\begin{tabular}{lccc}
\hline & $\begin{array}{c}\text { Cataract } \\
\text { (without diabetes) }\end{array}$ & $\begin{array}{c}\text { Cataract } \\
\text { (with NPDR) }\end{array}$ & $\begin{array}{c}\text { Cataract } \\
\text { (with PDR) }\end{array}$ \\
\hline $\begin{array}{l}\text { Preoperation } \\
\text { Postoperation }\end{array}$ & $6.94 \pm 0.34$ & $7.03 \pm 0.27$ & $8.94 \pm 0.57$ \\
1d & $26.27 \pm 1.37$ & $26.07 \pm 0.83$ & $32.42 \pm 0.67$ \\
$7 \mathrm{~d}$ & $13.96 \pm 1.05$ & $14.08 \pm 0.54$ & $19.86 \pm 0.78$ \\
30d & $9.07 \pm 0.43$ & $9.86 \pm 0.33$ & $13.24 \pm 0.29$ \\
90d & $7.16 \pm 0.27$ & $7.24 \pm 0.67$ & $11.25 \pm 0.31$ \\
\hline
\end{tabular}


in stage IV or $\mathrm{V}$, which may reduce the central visual acuity.

Interestingly, we found that there was a significantly higher flare value in the patients who had iris prolapse during the surgery at days 1 and 7 postoperatively. It suggests that gentle manipulation should be endured to alleviate the disorders of the BAB.

Since a significant correlation was found between lens epithelial cell proliferation and postoperative BAB breakdown, ${ }^{10}$ strategies to minimize the disorders of the $\mathrm{BAB}$ must be studied further.

\section{References}

1 Sanders DR, Kraff MC, Liebermann HL, Peyman GA, Tarabishy S. Breakdown and reestablishment of blood-aqueous barrier with implant surgery. Arch Ophthalmol 1982; 100: 588-590.

2 Shah SM, Spalton DJ. Changes in anterior chamber flare and cells following cataract surgery. Br J Ophthalmol 1994; 78: 91-94.

3 Alio JL, Sayans JA, Chipont E. Flare-cell meter measurement of inflammation after uneventful cataract surgery with intraocular lens implantation. J Cataract Refract Surg 1997; 23: 935-939.
4 Schauersberger J, Kruger A, Mullner-Eidenbock A, Petternel V, Abela C, Svolba G et al. Long-term disorders of the bloodaqueous barrier after small-incision cataract surgery. Eye 2000; 14: 61-63.

5 Saari KM, Guillen-Monterrubio OM, Hartikainen J, Hamalainen MM, Taskinen K. Measurement of protein concentration of aqueous humour in vivo: correlation between laser flare measurements and chemical protein determination. Acta Ophthalmol Scand 1997; 75: 63-66.

6 Mester U, Strauss M, Grewing R. Biocompatibility and blood-aqueous barrier impairment in at-risk eyes with heparin-surface-modified lenses. J Cataract Refract Surg 1998; 24: 380-384.

7 Pande MV, Spalton DJ, Kerr-Muir MG, Marshall J. Postoperative inflammatory response to phacoemulcification and extracapsular cataract surgery:aqueous flare and cells. J Cataract Refract Surg 1996; 22 (Suppl 1): 770-774.

8 Miyake K, Ota I, Miyake S, Maekubo K. Correlation between intraocular lens hydrophilicity and anterior capsule opacification and aqueous flare. J Cataract Refract Surg 1996; 22 (Suppl 1): 764-769.

9 Oshika T, Suzuki Y, Kizaki H, Yaguchi S. Two year clinical study of a soft acrylic intraocular lens. J Cataract Refract Surg 1996; 22: 104-109.

10 Pande MV, Spalton DJ, Marshall J. In vivo human lens epithelial cell proliferation on the anterior surface of PMMA intraocular lenes. $\mathrm{Br}$ J Ophthalmol 1996; 80: 469-474. 